\title{
Shock-strength determination with seeded and seedless laser methods
}

\author{
G. C. Herring and James F. Meyers \\ NASA Langley Research Center, Hampton, VA 23681-2199, USA
}

\begin{abstract}
Two nonintrusive laser diagnostics were independently used to demonstrate the measurement of time-averaged and spatially-resolved pressure change across a twodimensional (2-D) shock wave. The first method is Doppler global velocimetry (DGV) which uses water seeding and generates 2-D maps of 3-orthogonal components of velocity. A DGV-measured change in flow direction behind an oblique shock provides an indirect determination of pressure jump across the shock, when used with the known incoming Mach number and ideal shock relations (or Prandtl-Meyer flow equations for an expansion fan). This approach was demonstrated at Mach 2 on 2-D shocks and expansions generated from a flat plate at angles-of-attack $\approx-2.4^{\circ}$ and $+0.6^{\circ}$, respectively. This technique also works for temperature jump (as well as pressure) and for normal shocks (as well as oblique). The second method, laser-induced thermal acoustics (LITA), is a seedless approach that was used to generate 1-D spatial profiles of streamwise Mach number, sound speed, pressure, and temperature across the same shock waves. Excellent agreement was obtained between the DGV and LITA methods, suggesting that either technique is viable for noninvasive shock-strength measurements.
\end{abstract}

Key Words: Oblique shock strength, Prandtl-Meyer expansion, Doppler global velocimetry (DGV), Laser-induced thermal acoustics (LITA)

PACS: $\quad 00.06,00.07$ 


\section{Introduction}

\subsection{Background}

Four decades ago, after the invention of the laser, researchers began measuring off-body flow velocities in fluids by seeding various types of small particles into the flow and measuring the Doppler shift of the Mie-scattered signals [1, 2]. Historically, particleseeded techniques have measured only velocity, but not other flow parameters (with rare exception [3]). Other diagnostics are required if data beyond velocity are needed. Hence, much effort has been spent developing scattering and fluorescence techniques that directly probe the flow molecules to provide additional information. These molecular techniques are typically complicated, expensive, and labor-intensive compared to particle methods. If pressure and/or temperature could be derived from particle-based methods, it would greatly enhance the value of particle methods to aeronautics research.

Significant research [4] is currently focused on reducing sonic-boom strengths, in order to demonstrate the feasibility of commercial cross-continental supersonic flights. Pressure-signature measurement in wind tunnels has long been an important aspect of ground testing, and has traditionally been accomplished with pressure probes. However, the influence of geometry on the probe data is a concern. Thus, noninvasive optical pressure measurement has the potential to improve the quality of ground-test efforts, including shock-strength measurement.

\subsection{Present Accomplishments}

This paper discusses two independent and noninvasive methods for measuring shock strength. The first, Doppler global velocimetry (DGV), uses one laser to determine static pressure at multiple off-body locations in a two-dimensional (2-D) sheet. Pressure is inferred from the flow-turning angle, which is derived from the spatially-resolved, 2-D maps of DGV flow velocity. This approach is practical for only a few special flow fields, where other information about the flow is known (e.g., the presence of shock waves). Particle image velocimetry (PIV) and Doppler anemometry (LDA) should work as well as DGV with this approach, but only if larger seed particles than the $\sim 1$-nm condensed water particles [5] of the present work are used. The present work concentrates on velocity and pressure jumps across shocks and expansions, using DGV.

The second method, laser-induced thermal acoustics (LITA), uses three crossed laser beams and provides an off-body point measurement, where the point is defined by the beam overlap volume. LITA is a seedless approach for directly measuring Mach number, static temperature, and static pressure [6]. It was used to produce 1-D traces over the same flows as in the above DGV work, but without introducing water as a seed. This allowed a comparison between two nonintrusive, spatially-resolved, and timeaveraged optical measurement techniques. Because simultaneous installation of both of the optical setups was impractical, the DGV and the LITA measurements were made sequentially, separated by 3 months. Thus the two measurements were made on nearly 
identical flow fields only to within the repeatability of the wind tunnel operation and model positioning.

\section{Description of Experiments}

\subsection{DGV Velocity Method}

DGV is used for off-body flow velocimetry, and descriptions of this technique can be found in the literature [2, 7-11]. A single-longitudinal frequency of an Ar-ion cw laser is tuned part way up the side of an $\mathrm{I}_{2}$ absorption feature near $514 \mathrm{~nm}$. The laser is actively locked to the side of the $\mathrm{I}_{2}$ transition to stabilize the frequency drift of the laser. This is accomplished by passing a small fraction of the laser beam through a vaporlimited $\mathrm{I}_{2}$ vapor cell (the first of two in the setup) and using the observed absorption signal in a feedback loop to control the laser frequency. The remaining majority of the laser beam power is formed into a thin sheet to define a planar interrogation region. Rayleigh scatter from ice particles passing through the laser sheet is imaged onto three video cameras observing from three different directions. These three different scattered signals exhibits distinct Doppler shifts, since the Doppler shift of the scatted light depends on the observation direction as well as flow velocity.

The three DGV optical signals are passed through a second temperaturestabilized, vapor-limited $\mathrm{I}_{2}$-vapor cell, before being directed to the video cameras. Each of the three DGV signals, with its unique Doppler shift, undergoes a different absorption magnitude as it transits this second $\mathrm{I}_{2}$ cell. Thus, the measured signal intensity on each camera can be related to frequency, which in turn is related to Doppler shift and, finally related to flow velocity. Optimal choice of three non-orthogonal observation directions allows determination of three orthogonal components of the total velocity vector. In this manner, three components of flow velocity are measured simultaneously, at multiple points in the plane defined by the light sheet. The spatial resolution is determined by the magnification of the collection optics and the camera properties.

In the present DGV work, typically 3 liters of liquid $\mathrm{H}_{2} \mathrm{O}$ is added to the wind tunnel circuit, until the stagnation-chamber humidity reaches a dew point of about $-12{ }^{\circ} \mathrm{C}$. This generates a fog of tiny ice crystals, in the cold freestream test section flow, that increases the scattered light level. DGV velocity measurements were made in a series of streamwise positions, with respect to a flat plate, with a cross-flow light sheet oriented orthogonal to the freestream flow direction. All DGV measurements presented here were averaged over $\approx 1 \mathrm{sec}$. The spatial resolution in a single planar velocity map is $\approx 0.2 \mathrm{~mm}$. Additional details of the DGV test have been described previously [10].

Repeated DGV measurements of the streamwise freestream velocity over constant facility conditions, within a single day, show about $0.2-0.4 \%$ fluctuations. These small deviations may show the limit of the DGV instrument and/or flow repeatability. Furthermore, from day-to-day, 5 times larger deviations, of 1-2\% in freestream velocity, were observed. It is has not yet been determined whether the $1-2 \%$ 
day-to-day deviations are from uncertainties in the DGV instrument or the humidity measurement, or true tunnel velocity variations.

\subsection{DGV Pressure-Jump Method}

The present paper describes a novel demonstration for the determination of the pressure jump across a shock wave, based on a DGV-measured velocity vector map. A two-step process, using the (1) oblique and (2) normal shock relations, is used to convert the velocity data to pressure data. If a DGV velocity map spans an oblique shock, then the flow-turning angle behind the shock can be determined. Once the change in direction of the flow velocity is measured, the ideal shock relations (found in most aeronautics textbooks, e.g., [12]) are used to calculate the change in pressure across the shock.

More specifically, the direct DGV velocity measurement in front and behind the shock gives the angular change $\delta$ in flow direction across the shock. The oblique-shock relations, with incoming freestream Mach number $\mathrm{M}_{1}$ and $\delta$, then determine a unique shock angle $\theta$. A known $\theta$ then determines (again from the oblique-shock relations) the change in Mach number component that is normal to the shock. Finally, the normalshock relations are used to determine the pressure jump across the shock. With the present small data set, pressure jumps were determined by simply reading numbers from shock tables. However, to analyze larger data sets in the future, automation of this process will require a few iterative computer algorithms.

Measurement of the temperature jump across the shock is equally feasible, with the same approach as for pressure. In addition, both pressure and temperature decreases across expansion fans are also obtainable, by an analogous method. For expansions, $\mathbf{M}_{1}$ is used with the measured flow-turning angle $\delta$ to determine the Mach number $\mathrm{M}_{2}$ behind the expansion with the ideal Prandtl-Meyer flow equations (also in most textbooks [12]). Then $\mathrm{M}_{1} / \mathrm{M}_{2}$ determines the pressure and temperature changes according to the isentropic flow relations (also in most textbooks [12]).

\subsection{LITA Method}

LITA is one of a myriad of laser techniques that measure fluid properties by directly probing the molecules that make up the flow medium - usually without seeding. In most of the present LITA tests, water seeding was not used, and the tunnel dew point was about $-35^{\circ} \mathrm{C}$. Detailed descriptions of LITA-velocimetry development at NASA Langley Research Center (LaRC) can be found elsewhere for supersonic [6], transonic [13], and subsonic [14] flows, while the work of other groups is summarized in a recent review [15].

In LITA, two focused and crossed 1.06- $\mu \mathrm{m}$ laser beams from a Q-switched $\mathrm{Nd}$ : YAG laser (150 mJ per pulse per beam) induce two counter-propagating sound-wave packets in the gas. These sound waves, or gas-density gratings, have a sound pressure level of $\sim 100 \mathrm{~dB}$ (re $20 \mu \mathrm{Pa}$ ), i.e., a fractional density change of $\sim 10^{-4}$. Thus the technique can be characterized as nonintrusive. A third laser beam (probe at $532 \mathrm{~nm}$ ) 
intercepts the sound wave packets, which reflect a tiny fraction of the incident probe to a detector positioned at the Bragg-scattering angle. Mach number and sound speed are determined from Doppler shifts of the signals; Mach number does not require calibration.

LITA measures sound speed and the streamwise component of Mach number simultaneously (and thus velocity) in the overlap volume defined by the three laser beams crossed at a small angle, typically $~ 1 \mathrm{deg}$. If the flow composition is known, then translational temperature (not rotational or vibrational) can be derived from the sound speed, and gas pressure is measurable under certain restricted circumstances $[6,16]$. The LITA-inferred Mach number, sound speed, and temperature are derived from MHzmodulations in the light scattering from the LITA-induced sound waves. The pressure information is derived from the observed absorption of the induced sound waves, as described in reference 6. All LITA measurements presented here have an ellipsoidal shaped sample volume of $0.5 \mathrm{~mm}$ by $0.5 \mathrm{~mm}$ by $1 \mathrm{~cm}$ (oriented perpendicular to the freestream direction) and are time averaged over $17 \mathrm{sec}$ (500 laser pulses at a $30 \mathrm{~Hz}$ rate). All measurements are made about 5-8 cm above the model centerline.

Details of the specific LITA apparatus used in the present discussion have been given previously [6], where it was demonstrated to provide measurements of static pressure $( \pm 4 \%)$ and temperature $( \pm 0.4 \%)$, sound speed and one component of velocity $( \pm 0.2 \%)$, without seeding water particles into the Unitary Plan Wind Tunnel (UPWT) flow. These freestream uncertainties provide estimates of the limitation the present LITA instrument (except for pressure as discussed in section 4.4). The short LITA test duration did not allow the accumulation of many day-to-day measurements of the streamwise freestream velocity.

\subsection{Wind Tunnel and Flat-Plate Model}

All measurements were made in test-section 1 of the closed-circuit variableMach-number UPWT at LaRC. A flat-plate model was installed in the 1.2 by 1.2-meter test section and used to generate 2-D oblique shock or expansion waves. Additional details of the UPWT [17] and the flat-plate model [11] are available. The flat plate was oriented at two nominal angles-of-attack (AOA), -3 and 0 deg, for measurements with both of the instruments. The nominal AOA were calibrated with an inclinometer that was mounted on the sting (not the plate). The centerline of the model is approximately collinear with the tunnel centerline. All of the present work was performed in air at Mach 2 (velocity $=535 \mathrm{~m} / \mathrm{sec}$ ), a free-steam static pressure of $7.6 \mathrm{kPa}$, and a freestream static temperature of $183 \mathrm{~K}$. A schematic of the location of both the DGV and LITA optical measurements, relative to the model, is shown in the side-view of figure 1 . The location of the horizontal line indicating the LITA measurements is roughly on both the centerlines of both the tunnel and the model. A photograph of the flat-plate model is shown in figure 2, with a store located slightly off of the model centerline.

Although the DGV and LITA measurements were made sequentially, the model was not removed from the test section between the two tests. Thus the AOA was expected to be reproducible for the two tests, to within the repeatability of the model 
support apparatus. This expectation was realized, based on the DGV and LITA measurements that are described below. However, the nominal AOA of -3 and 0 deg were found (by both optical methods) to be actually $\approx-2.4$ and $+0.6 \mathrm{deg}$, respectively, and these observed values will be referred to for the remainder of this discussion.

\section{Measurement Results}

Measured flow parameters by both methods include the streamwise freestream velocity $V_{1}$ in front of the shock, the velocity $V_{2}$ behind the shock, as well as the computed differences $\Delta \mathrm{V}=\mathrm{V}_{2}-\mathrm{V}_{1}$ and ratios $\mathrm{V}_{2} / \mathrm{V}_{1}$. The quantities $\Delta \mathrm{P}$ and $\delta$ are the pressure change and flow-turning angle across the shock or expansion wave. $T_{1}$ and $T_{2}$ are temperatures, before and after the oblique waves, respectively. Note that $\delta=-\mathrm{AOA}$ for an ideal 2-D oblique shock from a wedge or flat plate.

\subsection{DGV}

Figure 3 shows an upstream view of two 2-D vertical cross sections of DGVmeasured velocity: the left and right side for the -2.4 and +0.6-deg AOA conditions, respectively. Color represents the streamwise velocity and is calibrated with the color scale shown. The direction of the cross-flow components is shown by the small arrows with the magnitude calibrated by the length of the reference arrow. For -2.4 deg AOA, it is seen that the freestream flow (i.e., above the horizontal shock) slows down and turns upward after it crosses the shock (i.e., below the shock). For +0.6 deg AOA, the streamwise flow is seen to speed up slightly just below the horizontal feature (green) and to slightly turn downward. Thus the data illustrates an expansion behind the leading edge (LE), not an ideal Mach wave as expected for a nominal 0-deg AOA.

Since the DGV data is a vertical slice through the shock, only the velocities just above and just below the shock should be used to identify $V_{1}$ and $V_{2}$ and the associated shock strength. In addition, only velocities from a single vertical column of pixels near the shock and model centerlines are used for comparison to the centerline LITA data. These DGV results are listed in table 1 for comparison to LITA.

Figure 3a clearly shows the vertical location of the shock. Similar data sets at other downstream locations, allow DGV to directly measure the shock angle $\theta$, as shown in figure 4. This determination of $\theta$ is independent from the method described above (that derives $\theta$ from the measured flow-turning angle $\delta$ and shock tables) and can be used to visualize the wave angles. One data point has been arbitrarily omitted from each linear fit of figure 4. The uncertainty of the illustrated linear fits is about $\pm 0.03 \mathrm{deg}$. It has not yet been determined whether the nonlinearity of the data is due to errors in the DGV measurements or shows real curvature of the oblique waves.

\subsection{LITA}

LITA measurements of streamwise Mach number $\mathrm{M}$, temperature, and pressure are shown for -2.4 deg AOA in figure 5 and for +0.6 deg AOA in figure 6. For -2.4-deg, 
one sees the Mach number drop (with associated temperature and pressure jumps) across the oblique shock. LITA values of Mach number and temperature are combined to compute velocity and are listed in table 1 to compare with the DGV measurements. For +0.6 deg AOA, an expansion fan is seen by the Mach number increase over the $10-20 \mathrm{~cm}$ region (with associated temperature and pressure drops). Thus LITA and DGV both show an expansion fan, not a Mach wave, for the +0.6-deg AOA case.

In the LITA profiles of figure 6 , a "micro shock" (located at $\approx 8 \mathrm{~cm}$ ) is seen just before the start of the expansion fan. This micro shock is also seen in the DGV data, as a green horizontal stripe at vertical location $70 \mathrm{~mm}$, above the surface, in figure 3b. Again DGV and LITA agree well, by showing similar fine details for the same flow field. This micro shock is probably generated by the finite radius of curvature of the sharp LE of the plate, which essentially provides a short portion of LE that has a negative AOA - even if the plate has an overall positive AOA. Another possibility is that the micro shock is generated from the growing boundary layer on the forward portion of the plate. Henceforth, this micro shock is referred to as the LE shock.

In figures 5a and 6a each, two profiles of LITA measurements of Mach number are shown. Data connected with solid lines are from a dry run (no water seeding) with dew point $=-35^{\circ} \mathrm{C}$, and data connected with dashed lines are from a humid run (using water seeding) with dew point $=-12{ }^{\circ} \mathrm{C}$ (the same as that used during the DGV work in the above discussion). First, note that the freestream data, in front of the shocks, show the expected effect [18] of water seeding, i.e., a decrease of $\Delta \mathrm{M}=0.05$ from gas heating due to condensation of water vapor to ice crystals in the expansion of the nozzle. Second, note that dry and humid Mach-number data show the same change $\Delta \mathrm{M}$ for both shocks in both figure 5a and 6a. Agreement between dry and humid $\Delta \mathrm{M}$ shows that shock-strength measurements of DGV (with high humidity) are not significantly different from those in dry conditions for the present work.

\subsection{Velocity Jump $V_{2}-V_{1}$}

Measurements are summarized in table 1, showing a comparison of DGV and LITA made at the crossing point of the dotted lines in figure 1. The top row corresponds to the -2.4 deg AOA, while the bottom row corresponds to +0.6 deg AOA. Columns 1 and 2 show DGV measurements of the ratio and difference velocities, while columns 3 and 4 show the comparable LITA values. DGV values are extracted from the streamwise velocity data of the two images of figure 3 (left side of figure $3 a \Rightarrow$ top row of table 1 and right side of figure $3 \mathrm{~b} \Rightarrow$ bottom row). In each case, the velocity ratio and difference was determined from a single column of pixels from the approximate central region of the image. LITA values were obtained from figure 5 and 6. Negative values for the difference $V_{2}-V_{1}$ in the top row indicate that the streamwise flow slowed behind the shock wave. Positive values of the bottom row indicate that the flow behind the expected Mach wave actually speeds up. Both techniques clearly show that the plate was tilted slightly upward, resulting in an expansion fan behind the LE, for the +0.6 deg AOA case.

\subsection{Flow-Turning Angle $\delta$}


Column 1 of table 2 shows DGV-measured flow-turning angle $\delta$ for both AOA, while column 3 shows the LITA-inferred values. DGV values are computed from $\delta=$ $\arctan \left(\mathrm{V}_{2}\right.$ transverse $\left./ \mathrm{V}_{1 \text { streamwise}}\right)$, using the appropriate measured velocity components in front and behind the shock or expansion wave. In column 3 , the LITA $\delta$ is determined indirectly with an adjustable $\delta$ that is fit to the measured streamwise Mach numbers (in front and behind the shock) and constrained by the oblique shock relations (or PrandtlMeyer relations in the case of an expansion). The $\delta$ values of 2.2 and 2.5 from the top row of table 2 were averaged to get $\mathrm{AOA}=-\delta \approx-2.4$ (shown in column 5 of table 2), while the values of -0.4 and -0.7 for $\delta$ from the bottom row were averaged to AOA $\approx$ +0.6 deg in column 5). Positive signs for $\delta$ indicate that the streamwise flow is turned upward in figure 1 , while negative signs indicate a downward turn for the flow.

Column 2 of table 2 shows the shock angle $\theta$ as measured by DGV in the data of figure 4. This $\theta$ measurement (described in section 3.1) is distinct and independent from the $\theta$ derived from measured flow-turning angle $\delta$ and the shock relations (in the process of determining pressure jumps $\Delta \mathrm{P}$ as described in section 2.2). However, the two sets of $\theta$ are in good agreement. Column 4 is empty since the LITA data of figure 5 and 6 are acquired at only one vertical position, and thus LITA was not capable of directly illustrating the shock angle with the present data sets.

Both optical techniques independently determined that the -3 deg nominal AOA was slightly greater than -3 deg, giving a shock wave slightly weaker than expected. Both techniques also showed that the 0 -deg nominal AOA was actually a slightly positive angle, giving an expansion wave instead of an ideal Mach wave. For each AOA, the DGV-measured AOA was in agreement with the LITA-inferred AOA, to within the uncertainties estimated below in section 4.4.

\subsection{Pressure Jump $\triangle P$}

Column 1 of table 3 shows the first of two main results of this paper, which is the DGV-derived pressure jump $\Delta \mathrm{P}$ of $0.91 \mathrm{kPa}$ across the shock wave in the top row (or drop for the value $-0.40 \mathrm{kPa}$ of the expansion wave in the bottom row). Columns 2-4 show the second main result, which is the LITA-derived pressure jumps. The top row contains results for -2.4 deg AOA, while the bottom row contains the corresponding values for $\mathrm{AOA}=+0.6$ deg. LITA values are derived from the data of figures $5 \mathrm{a}$ through 6c, while the DGV values are derived from the data of figure 3.

Pressure jump can be derived from LITA by any of three different methods. The column-2 values (superscript M) come from Mach-number data of figures 5a and 6a, while the column-3 values (superscript $T$ ) come from the temperature data of figures $5 b$ and 6b. Last, the column-4 values (superscript P), directly-measured pressure jumps, come from figures $5 c$ and $6 c$. The jump in Mach number $M$ (figures $5 a$ and $6 a$ ) is unambiguous, with little variation at locations behind the shock and suggests that $\Delta \mathrm{P}^{\mathrm{M}}$, derived from $\mathrm{M}$ data, is the best of the three LITA methods. But for temperature and 
pressure, there is "apparent" significant downstream noise in the spatial profiles of $\mathrm{T}$ and $\mathrm{P}$ in figure 5. In the analysis for this paper, the apparent downstream fluctuation is ignored and only the initial jump in $\mathrm{T}$ or $\mathrm{P}$ is used to estimate $\Delta \mathrm{P}^{\mathrm{T}}$ and $\Delta \mathrm{P}^{\mathrm{P}}$.

\section{Discussion}

A qualitative description of the expectations and observations, for the two flow fields, is summarized in the last four columns of table 2. The top and bottom rows show, respectively, the -2.4 and +0.6 deg AOA conditions. All expected values from tables 1-3 are calculated starting with the expected AOA of column 7. These expected values are to be compared with the corresponding measured values from DGV or LITA in each table.

\subsection{Velocity Jump}

The good agreement of $~ 1$ part in 1000 between the two techniques is obtained because table 1 reports only velocity ratios or differences (not the velocities $V_{1}$ or $V_{2}$ ). Only weak oblique waves were studied, thus the velocities in front and behind the wave are very close in magnitude. If either of the techniques were to contain a systematic error in velocity, one might expect that typical errors would be approximately the same (in front of and behind the wave) and hence roughly cancel in computed ratios or differences. On the other hand, if the uncertainties are random, then forming differences or ratios would increase the uncertainty, relative to that of a single measurement.

Negative values of $\Delta \mathrm{V}=\mathrm{V}_{2}-\mathrm{V}_{1}$ in the top row of table 1 show that the flow slows down by $\approx 10 \mathrm{~m} / \mathrm{sec}$ behind the shock. Positive values of $\mathrm{V}_{2}-\mathrm{V}_{1}$ (bottom row) indicate that the flow behind the expansion fan speeds up by $\approx 4 \mathrm{~m} / \mathrm{sec}$. A difference in $\Delta \mathrm{V}$ of $\approx 1 \mathrm{~m} / \mathrm{sec}$, between the methods, is $0.2 \%$ of the freestream velocity of $535 \mathrm{~m} / \mathrm{sec}$. This agreement between DGV and LITA for each of the two flows is impressive, considering that the two measurements were made months apart (but without removing the model from the sting). Although a small degree of this excellent agreement may be fortuitous, nevertheless it provides confidence in either of the techniques if they were to be used alone in future work to measure either velocity jumps or ratios.

\subsection{Flow-Turning Angle}

The two optical methods agree with each other better than they agree with the inclinometer calibration, which provides the expected AOA. In addition, the sum of the two expected AOA (3 deg) roughly equals the sum of the two observed AOA $(\approx 3.0 \mathrm{deg}$ from table 2). This is consistent with the idea that the relative AOA readings (based on the inclinometer calibration) should be more accurate than the absolute values - if the sting is regularly moved in the same direction for both the DGV and LITA tests to minimize backlash effects. Third, both optical methods independently provide a better qualitative method for measuring small AOA, because they clearly illustrate whether the flow speeds up or slows down behind the oblique wave. Thus both optical techniques appear as good as, if not better than, the current inclinometer method for determining AOA of the flat plate. 


\subsection{Pressure Jump}

The important contribution of this work is the demonstration of four different measurements (one from DGV and three from LITA) for the determination of staticpressure jump across shock and expansion waves. Three of these, the DGV $\Delta \mathrm{P}$ and two LITA methods $\left(\Delta \mathrm{P}^{\mathrm{M}}\right.$ and $\left.\Delta \mathrm{P}^{\mathrm{T}}\right)$, are indirect measures of pressure, since they all require the use of the ideal shock relations or Prandtl-Meyer expansion relations to infer pressure change. These three measurements agree well, with typical differences of $\approx 0.1 \mathrm{kPa}$ for pressure jump in both the top and bottom rows of table 3 . Thus, three of the four methods agree to within $\sim 1 \%$ when referenced to the nominal freestream static pressure of $7.6 \mathrm{kPa}$. As with velocity, this agreement provides confidence in either of the techniques if they were to be used alone in future work to measure pressure jumps.

The fourth method (i.e., the third LITA method $\Delta \mathrm{P}^{\mathrm{P}}$ ) provides a highly-desired direct measure of pressure jump. However, it is disappointing to see that it disagrees with the other three approaches. This may be related to the "apparently noisier" pressure profiles, seen in figures $5 \mathrm{c}$ and 6c. First, the extra noise associated with the pressure profiles and $\Delta \mathrm{P}^{\mathrm{P}}$ may really be noise and be related to the fact that $\Delta \mathrm{P}^{\mathrm{P}}$ is based on signal amplitude data, whereas $\Delta \mathrm{P}^{\mathrm{M}}$ and $\Delta \mathrm{P}^{\mathrm{T}}$ are derived only from frequency data. Frequency information is typically more accurately measured than amplitude information. Second, the noise may be due to the fact that flat-plate shocks in UPWT slightly wiggle with time (but at a slower rate than the present measurement integration times). This statement is based on unpublished data from video-based Schlieren systems. Third, this apparent noise may possibly be real spatial structure of the oblique shock wave. Fourth, the large fluctuation in pressure, if real, means that the full pressure jump may have been missed due to the coarse spatial resolution (less likely in the smoother $M$ profiles of figures $5 \mathrm{a}$ and 6a). Additional discussion of these speculative ideas is beyond the scope of this work, but clearly, higher spatial resolution would improve the measurements of figures 5 and 6.

In summary, for the LITA data, $\Delta \mathrm{P}^{\mathrm{M}}$ is superior to $\Delta \mathrm{P}^{\mathrm{T}}$ because the jump in $\mathrm{M}$ is clear, with minimal noise. In turn, $\Delta \mathrm{P}^{\mathrm{T}}$ is superior to $\Delta \mathrm{P}^{\mathrm{P}}$, because $\Delta \mathrm{P}^{\mathrm{T}}$ is derived from frequency data and $\Delta \mathrm{P}^{\mathrm{P}}$ is derived from amplitude data. If the uncertainty of $\Delta \mathrm{P}^{\mathrm{P}}$ can be reduced by 10 times, then LITA would provide accurate ( $\pm 0.005 \mathrm{kPa})$, nonintrusive, and direct shock-strength measurements. One possibility for reducing the $\Delta \mathrm{P}^{\mathrm{P}}$ uncertainty would be to use the same approach that has been used with pressure probes: simultaneous dual point measurements, where one point is located in front and one is located behind the shock. However, additional work must be done to fully understand the "apparent noise" on the pressure profiles of figures $5 c$ and 6c.

\subsection{Measurement Uncertainties}

One way to estimate the uncertainties in $\Delta \mathrm{P}$ or $\Delta \mathrm{V}$ is to use the observed differences in the two techniques (i.e., those shown in tables 1-3). This method gives an uncertainty that is akin to accuracy, not precision. However, the DGV measurements 
were made at dew point $=-12{ }^{\circ} \mathrm{C}$, while most LITA measurements were made at a drier condition, with a dew point of $-35^{\circ} \mathrm{C}$. Thus, estimating uncertainties in this way may be conservative, since some of the observed difference in the two measurements may be due the humidity difference [17] and not due to errors in the techniques.

A second, and better, way to estimate uncertainties is to use the freestream variations of each of the techniques (ignoring real tunnel fluctuations). This method gives an uncertainty that is more like precision, not accuracy. It is not possible to report good quality statistics for LITA with the limited data acquired in this short test. For LITA, rough estimates of the uncertainties can be made by observing the typical fluctuation in the freestream values in front of the shock or expansion waves of figures 5 and 6. Uncertainties of about $\pm 0.002(0.1 \%), \pm 1 \mathrm{~K}(0.5 \%)$, and $\pm 0.05 \mathrm{kPa}(0.7 \%)$ are estimated for Mach number, static temperature and static pressure, respectively, when inspecting the few freestream data points of figures 5 and 6 . These estimates may be more equivalent to upper limits, since the observed fluctuations may also include true facility temporal fluctuations.

For DGV, this second method of estimating the uncertainties in streamwise velocity can be found from the one-sigma standard deviations ( $1 \sigma$ or $68 \%$ confidence) in the observed spatial fluctuations of velocity over a single image $(\sim 10 \mathrm{~cm})$ of $0.2 \%$. By averaging over 50 images $(\approx 1 \mathrm{sec})$, one finds a temporal standard deviation of streamwise velocity of $0.05 \%$. These DGV statistical estimates of uncertainty [10] are more rigorous than those above, for LITA, and also are conservative, because they include true temporal or spatial variations of velocity over the flow field. Since multiple repetitions of $\Delta \mathrm{P}$ for a given shock are not available in this work, one reasonable approach to estimate the $\mathrm{DGV}$ pressure jump uncertainties is to compare the $\mathrm{DGV} \Delta \mathrm{P}$ to the LITA $\Delta \mathrm{P}$. Ignoring $\Delta \mathrm{P}^{\mathrm{P}}$, table 3 shows differences, and thus uncertainty estimates of about $0.1 \mathrm{kPa}$ for DGV.

The precision in $\delta$, as measured by LITA, is estimated from the manual fitting procedure used to determine the best-fit $\delta$ and is about \pm 0.3 deg. This uncertainty is consistent with the observed differences between LITA and DGG results for $\delta$ in table 2 .

\section{Summary}

Doppler global velocimetry (DGV) can be used for determining pressure change across shock waves by using measured velocity vector maps of the gas velocity and deducing the flow deflection behind the shocks. This approach was experimentally demonstrated in a Mach-2 flow with a flat-plate model, on 2-D oblique shock and expansion waves. Laser-induced thermal acoustics (LITA) was also used to independently obtain 1-D profiles of Mach number, static temperature and static pressure measurements across these same oblique flow fields. Both methods can also determine the model AOA.

Both DGV and LITA have been shown to exhibit uncertainties of $\sim 0.1 \%$ in velocity jump and $\sim 1 \%$ in pressure jump. Additionally, good agreement between the 
two nonintrusive methods was obtained for velocity and pressure change, across both compressions and expansions. Ignoring $\Delta \mathrm{P}^{\mathrm{P}}$, differences between the techniques were typically $0.2 \%$ for fractional velocity jump and $1 \%$ for fractional pressure jump (relative to the freestream values). The methods also typically agreed to within \pm 0.3 deg for AOA measurements. The good agreement of these two optical approaches provides confidence that each technique independently possesses good accuracy in the measurement of AOA, and velocity or pressure ratios across shock waves.

The DGV method was demonstrated for a restricted set of flows - oblique waves. However for these flow fields, it shows significant promise to provide 2-D velocity maps, along with static pressure and temperature jumps with a relatively uncomplicated apparatus and at relatively low cost per data point. On the other hand, LITA can measure velocity and pressure in a wide variety of flow fields, but is restricted to obtaining data at relatively few spatial points. This is because of the point nature and the associated higher cost of this technique. But, in principle, the seedless aspect of LITA provides a clear nonintrusive (and hence reliable) data set, and thus remains of interest in spite of its higher costs. The present work suggests that the combination of the two techniques provides a generally powerful aeronautics tool that would provide significant off-body velocity and pressure data with a high degree of confidence.

Valuable contributions of A. A. Cavone, M. T. Fletcher, R. C. Hart, M. A. Kulick, J. W. Lee, R. J. Mack, F. J. Wilcox, Jr., and the NASA LaRC UPWT staff to this work are gratefully acknowledged. 


\section{References}

[1] Yeh Y and Cummins H Z 1964 Localized fluid flow measurements with an He-Ne laser spectrometer Appl. Phys. Lett. 4 176-178

[2] Samimy M and Wernet M P 2000 Review of planar multiple-component velocimetry in high-speed flows AIAA Journal 38 553-574

[3] van Oudheusden B W, Scarano F, Roosenboom E W M, Casimiri E W F, and Souverein L J 2007 Evaluation of integral forces and pressure fields from planar velocity data for incompressible and compressible flows Exp. Fluids 43 153-162

[4] Aronstein D C and Schueler K L 2005 Two supersonic business aircraft conceptual designs, with and without sonic boom constraint J. of Aircraft 42 775-786

[5] Benedict L H, Griffith W C, Yanta W J, Spring III W C, and Boyd C F 1991 Quantification of Vapor Screen Analysis AIAA $22^{\text {nd }}$ Fluid Dynamics, Plasma Dynamics, and Lasers Conference, Honolulu, Hawaii (June 24-26) paper 91-1691

[6] Hart R C, Herring G C, and Balla R J 2007 Pressure measurement in supersonic airflow by differential absorptive laser-induced thermal acoustics Opt. Lett. 32 1689-1691

[7] Meyers J F, Lee J W, Schwartz R J 2001 Characterization of measurement error sources in Doppler global velocimetry Meas. Sci. Tech. 12 357-368

[8] Charrett T O H, Nobes D S, Tatam R P 2007 Investigation into the selection of viewing configurations for three-component planar Doppler velocimetry measurements Appl. Opt. 46 4102-4116

[9] Muller H, Eggert M, Czarske J, Büttner L, Fischer A 2007 Single-camera doppler global velocimetry based on frequency modulation techniques Exp. Fluids 43 223-232

[10] Christian W, Stockhausen, Klinner G J, Lempereur C, Barricau P, Loiret P, and Raynal J C 2007 Performance and accuracy investigations of two Doppler global velocimetry systems applied in parallel Meas. Sci. Tech. 18 2504-2512

[11] Meyers J F, Lee J W, Fletcher M T, Cavone A, Viramontes J A G 2006 Supersonic flow field investigations using a fiber-optic based doppler global velocimeter $13^{\text {th }}$ International Symposium on Applications of Laser Techniques to Fluid Mechanics Lisbon, Portugal, (June 26-29) Paper 1019

[12] John J E A 1984 Measurements in Compressible Flow Gas Dynamics (Boston: Allyn and Bacon, Inc.) (1 $1^{\text {st }}$ ed., Appendices A-D, pp. 371- 401)

[13] Herring G C, Hart RC, Balla R J, and Henderson B 2007 Prospects for nonlinear laser diagnostics in the jet noise laboratory NASA TM 2007-214893

[14] Hart R C, Herring G C, and Balla R J 2002 Common-path heterodyne laser-induced thermal acoustics for seedless laser velocimetry,” Opt. Lett. 27 710-712

[15] Brown M S, Li Y, Roberts W L, and Gord J R 2003 Analysis of transient-grating signals for reacting-flow applications Appl. Opt. 42 566-578

[16] R. Stevens and P. Ewart 2004 Single-Shot Measurement of Temperature \& Pressure Using Laser-Induced Thermal Gratings With a Long Probe Laser Appl Phys. B 78 111-117

[17] Jackson C M, Jr., Corlett W A, and Monta W J 1981 Description and calibration of the langley unitary plan wind tunnel NASA TP-1905 
[18] Herring G C, 2008 Mach Number Measurement with laser and pressure probes in humid supersonic flow AIAA Journal 46 2107-2109 


\section{Figure Captions}

Figure 1 Side-view schematic of test setup, showing flat plate, ellipsoidal store, oblique shocks, and measurement locations for DGV (a 2-D sheet transverse to the freestream direction) and LITA (line collinear with the tunnel and model centerlines). AOA $=0$ in this view.

Figure 2 Photograph of model, with attached store, installed in the UPWT, in a nearly streamwise view that shows the sting and horizontal support.

Figure 3 Streamwise view of three-orthogonal components of DGV velocity data in a spatially-resolved 2-D sheet that is transverse to the freestream direction and located $100 \mathrm{~mm}$ downstream from the leading edge (vertical dotted line in figure 1). The (a) -2.4 and (b) +0.6-deg AOA data are on the left and right respectively. Color indicates streamwise velocity, and orientation and length of the small arrows indicates the small transverse velocity components.

Figure $4 \quad$ Vertical location of (a) shock and (b) expansion fan versus streamwise position from DGV (i.e., illustration of directly-measured oblique angle $\theta)$.

Figure 5 LITA measurements of (a) Mach number, (b) static temperature, and (c) static pressure along a streamwise oriented line (horizontal dotted line of figure 1) for the oblique shock wave (-2.4 deg AOA). In (a) both dry (solid line) and humid (dotted line) Mach number measurements are shown (acquired on different days).

Figure $6 \quad$ Similar to figure 5, but showing LITA measurements of (a) Mach number, (b) static temperature, and (c) static pressure for an oblique expansion wave (+0.6 deg AOA). In (a), both dry (solid line) and humid (dotted line) Mach-number measurements are shown. 
Table 1: Measured velocity ratio and difference $(\mathrm{m} / \mathrm{sec})$ from DGV and LITA across oblique shock (top row) and expansion (bottom row) waves

\section{DGV}

$\underline{\mathrm{V}_{2} / \mathrm{V}_{1}}$

0.979

1.0070
LITA

$\underline{\mathrm{V}_{2} / \mathrm{V}_{1}}$

0.976

1.0076

4

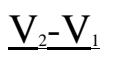

$-10$

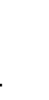

(1)

(1)

(1)

Table 2:

Measured flow-turning angle $\delta$, shock angle $\theta$, and AOA from DGV and LITA for shock (top) and expansion (bottom) waves [AOA $=-\delta]$

\begin{tabular}{|c|c|c|c|c|c|c|c|}
\hline \multicolumn{2}{|l|}{$\underline{\mathrm{DGV}}$} & \multicolumn{2}{|l|}{ LITA } & \multirow{2}{*}{$\begin{array}{l}\text { Observed } \\
\text { AOA } \\
\text { (deg) } \\
\end{array}$} & \multirow{2}{*}{$\begin{array}{l}\text { Observed } \\
\text { Flow }\end{array}$} & \multirow{2}{*}{$\begin{array}{l}\text { Expected } \\
\text { AOA } \\
\text { (deg) } \\
\end{array}$} & \multirow{2}{*}{$\begin{array}{l}\text { Expected } \\
\text { Flow }\end{array}$} \\
\hline $\begin{array}{l}\delta \\
(\mathrm{deg})\end{array}$ & $\begin{array}{l}\theta \\
(\mathrm{deg})\end{array}$ & $\begin{array}{l}\delta \\
(\mathrm{deg})\end{array}$ & $\begin{array}{l}\theta \\
(\mathrm{deg})\end{array}$ & & & & \\
\hline 2.2 & 34.5 & 2.5 & ----- & $\approx-2.4$ & $\begin{array}{l}\text { Oblique } \\
\text { Shock }\end{array}$ & -3 & $\begin{array}{l}\text { Oblique } \\
\text { Shock }\end{array}$ \\
\hline-0.4 & 34.8 & -0.7 & ----- & $\approx+0.6$ & $\begin{array}{l}\text { Oblique } \\
\text { Expansion }\end{array}$ & 0 & $\begin{array}{l}\text { Mach } \\
\text { Wave }\end{array}$ \\
\hline
\end{tabular}

Table 3: $\quad$ Measured pressure jump $\Delta P(\mathrm{kPa})$, from DGV and LITA, across oblique shock (top) and expansion (bottom) waves

\begin{tabular}{|c|c|c|c|c|}
\hline DGV & LITA & & & Expected \\
\hline$\Delta \mathrm{P}$ & $\Delta \mathrm{P}^{\mathrm{M}}$ & $\Delta \mathrm{P}^{\mathrm{T}}$ & $\Delta \mathrm{P}^{\mathrm{P}}$ & $\Delta \mathrm{P}$ \\
\hline 0.91 & 1.1 & 0.97 & 0.60 & 1.3 \\
\hline-0.20 & -0.29 & -0.25 & -0.46 & 0 \\
\hline
\end{tabular}




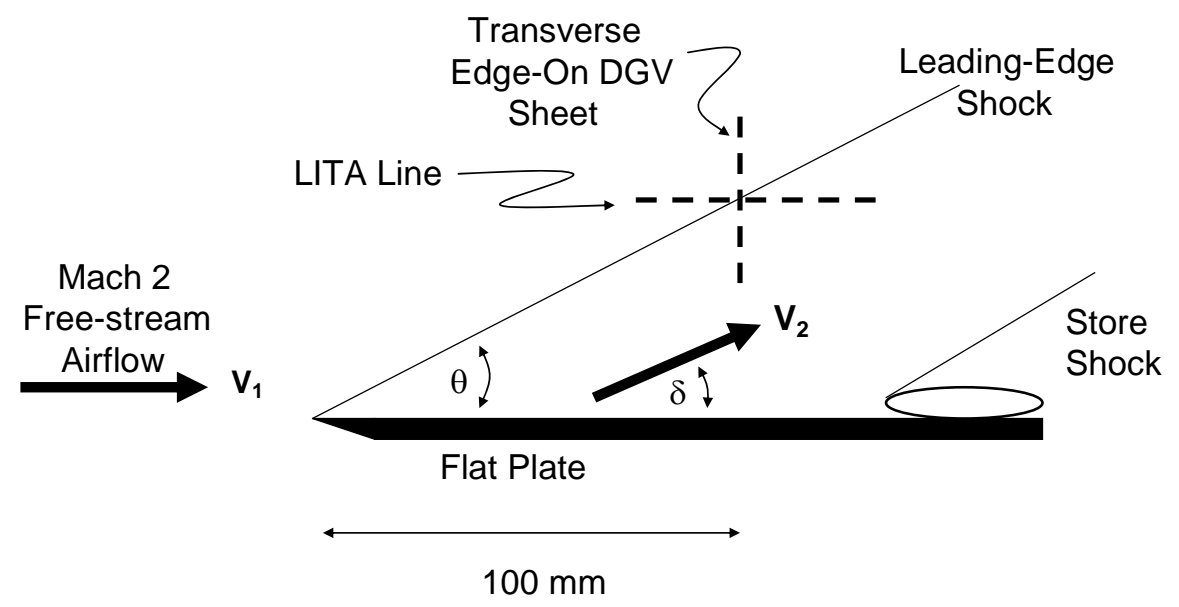

Figure 1

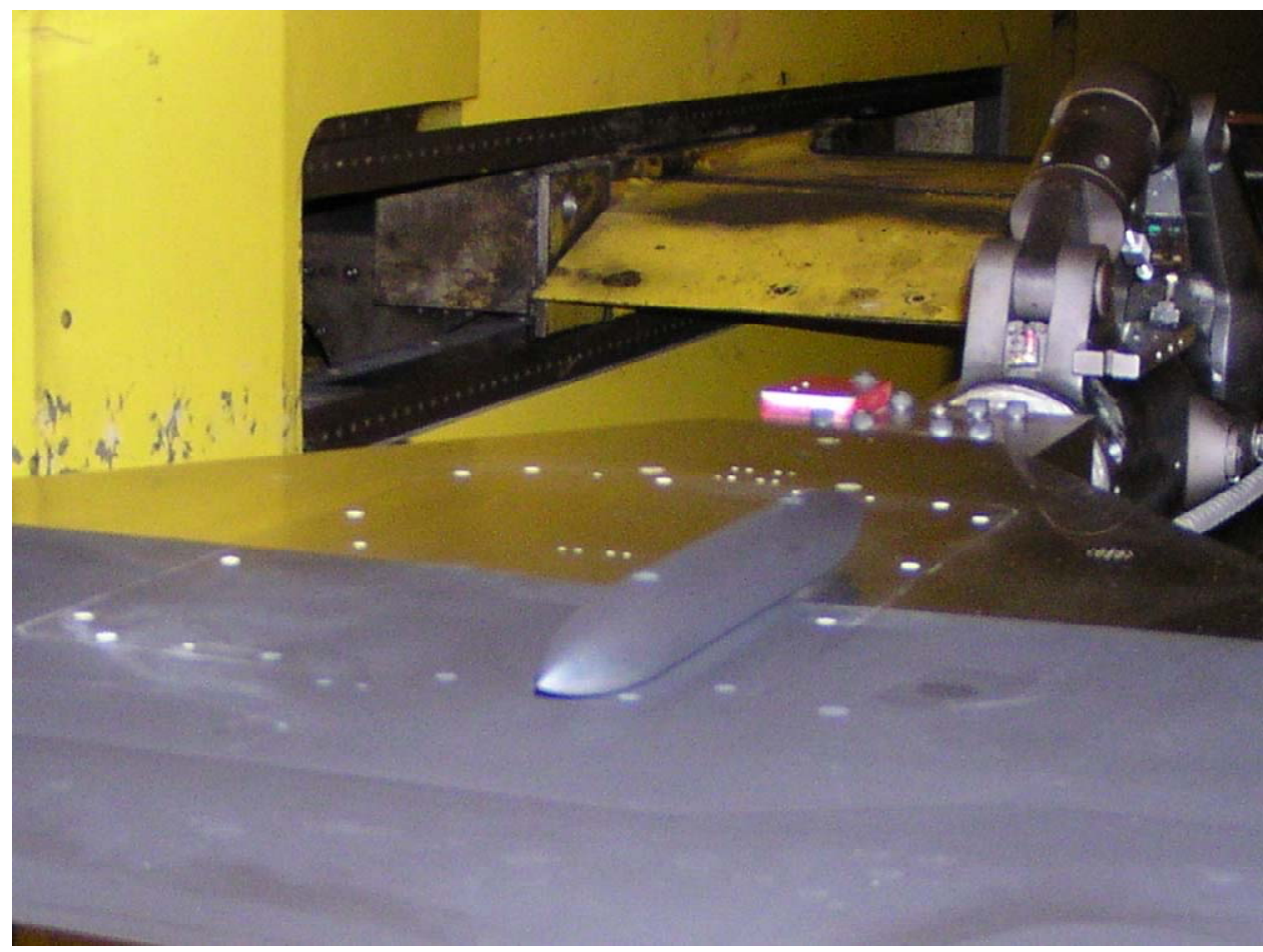

Figure 2 

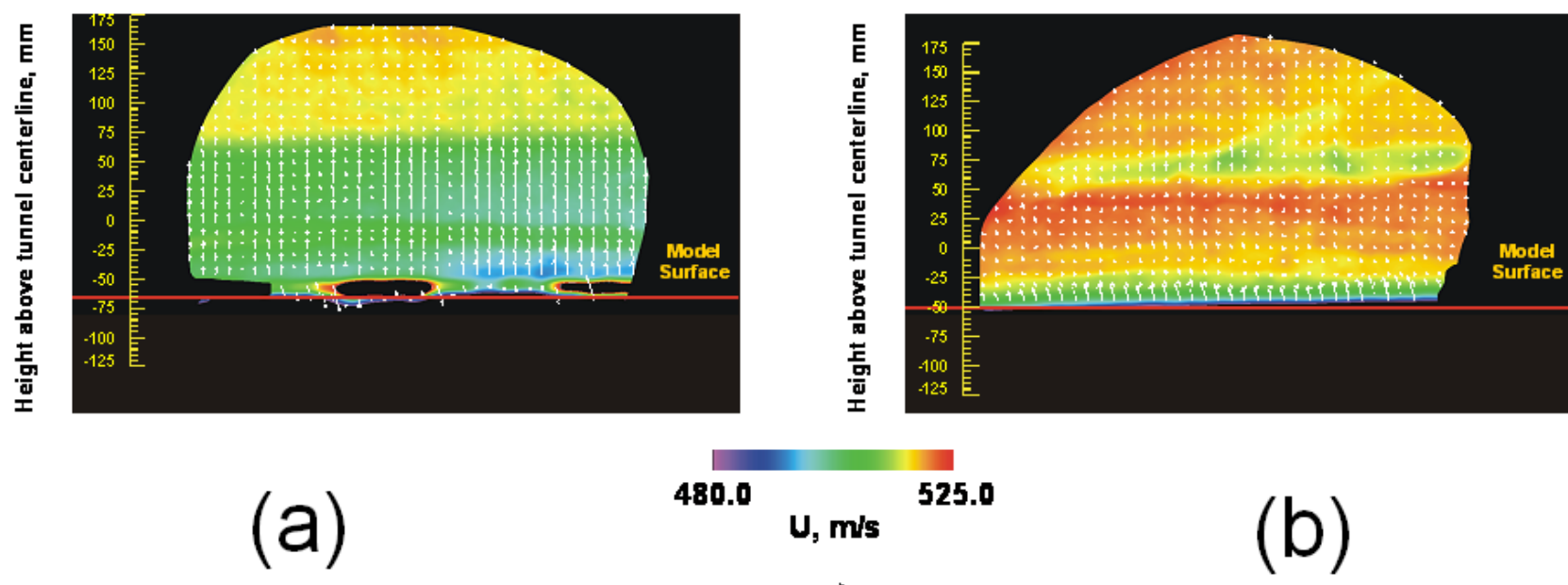

(b)

$30 \overrightarrow{\mathrm{m}} / \mathrm{s}$

Figure 3 - this figure should be large, two columns
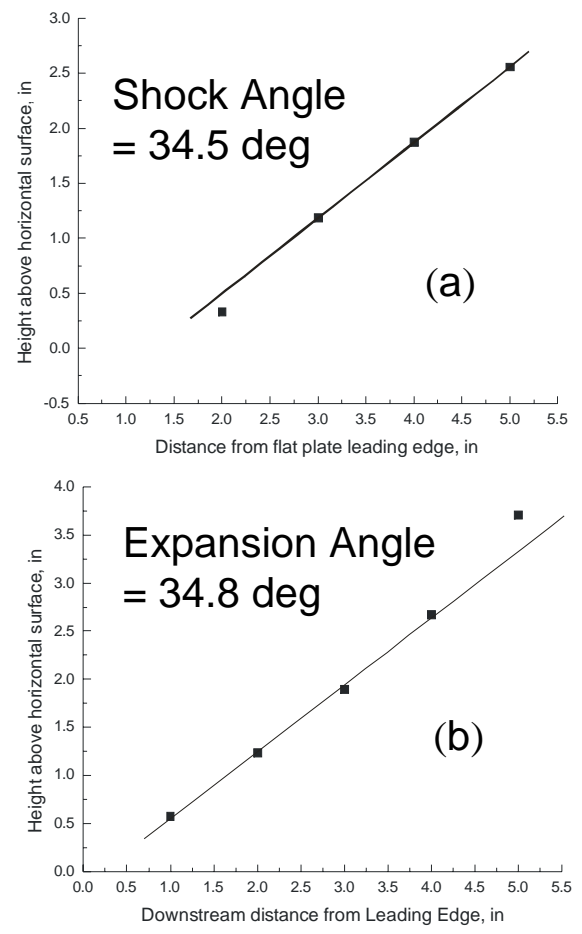

Figure 4 

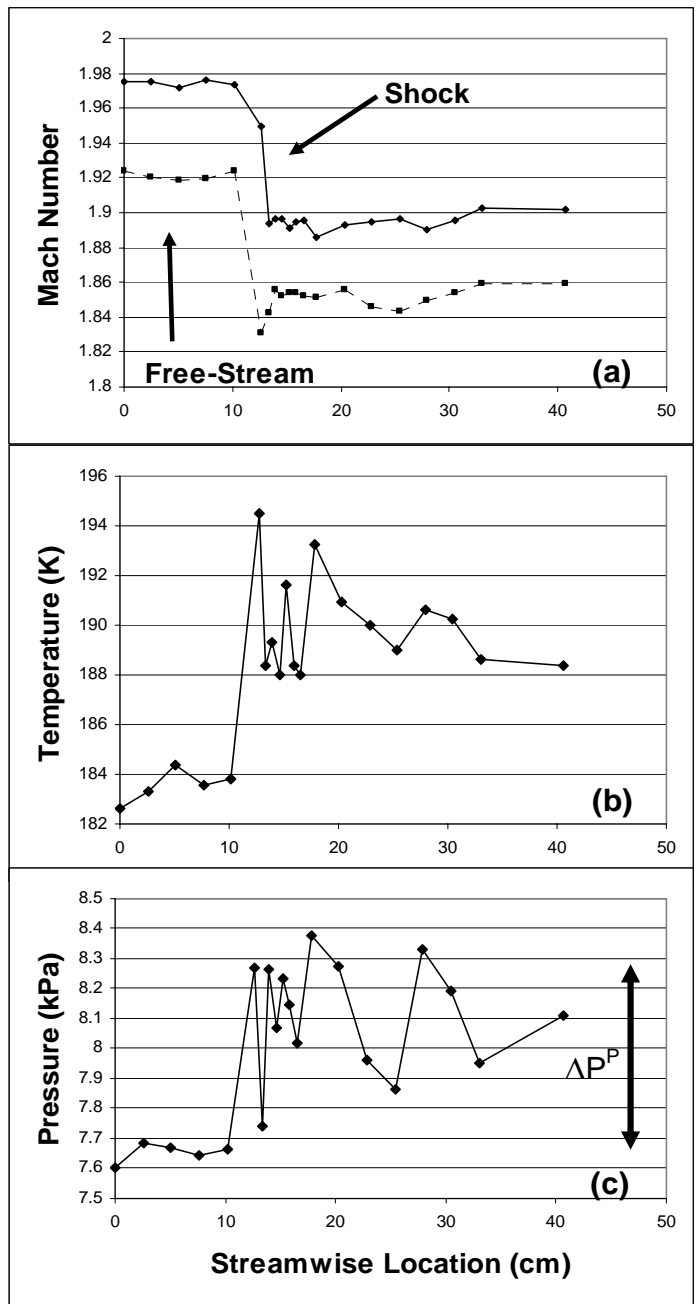

Figure 5 


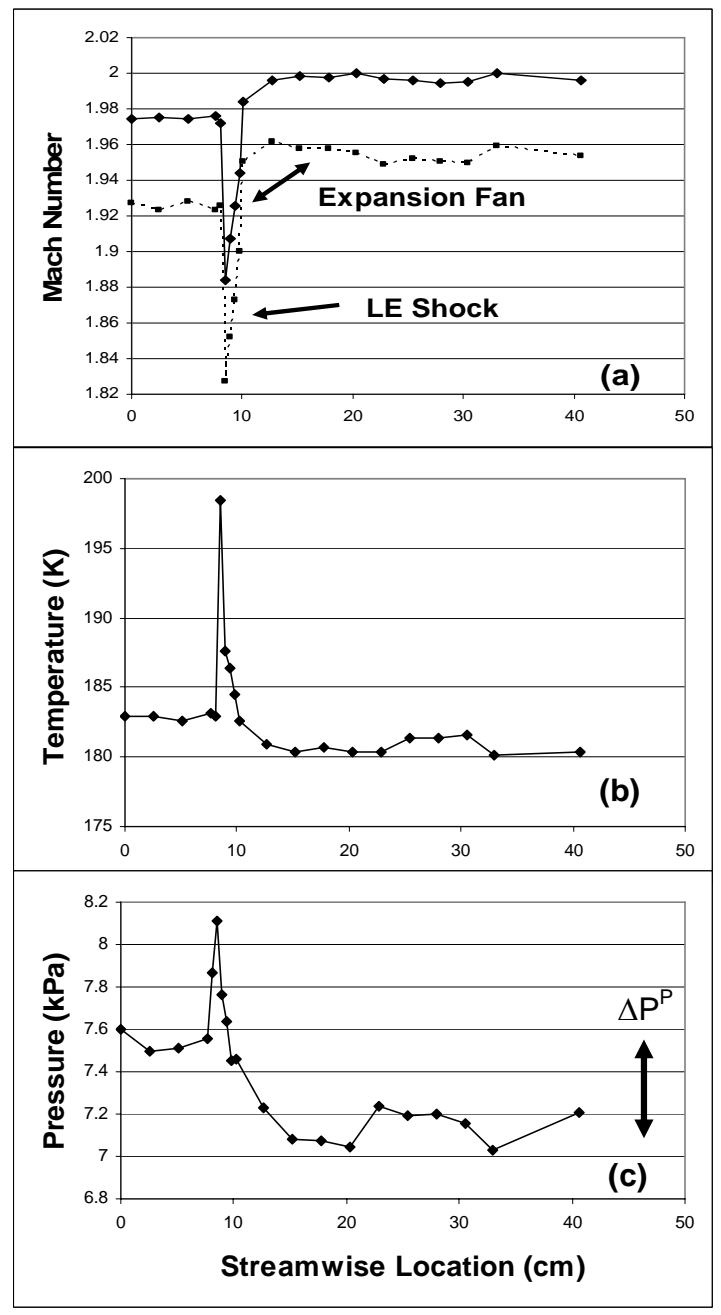

Figure 6 